\title{
¿Por qué un tratamiento precoz en la artritis reuma-
}

P. Benito Ruiz, A. Pros Simón

Servicio de Reumatología del IMAS.

Hospital del Mar. Hospital de la Esperanza.

Universidad Autónoma de Barcelona

\section{RESUMEN}

La artritis reumatoide es una enfermedad a utoinmune caracterizada por la inflamación crónica de las articulaciones diartrodiales, que además, puede ocasionar afectación de otros órganos. En la actualidad, la mayoría de autores están de acuerdo en que, a los 10 años de evolución, casi el $80 \%$ de los pacientes presenta algún grado de limitación. El pronóstico de la artritis reumatoide es incierto, y las lesiones, que se producen en los primeros años de enfermedad, pueden continuar a pesar de la mejoría clínica ya que la patogénesis de estas lesiones puede diferir de la simple inflamación aguda articular. El diagnóstico precoz es pues esencial para iniciar su tratamiento antes de que se produzca el daño anatómico articular, habiéndose demostrado que los pacientes tratados durante el primer año desde el comienzo de los síntomas mantienen una capacidad funcional mejor que los remitidos más tardiamente.

El tratamiento clásico de esta enfermedad había contemplado, hasta hace poco tiempo, una estructura piramidal, en la que de forma secuencial se iban introduciendo fármacos. Los pobres resultados obtenidos con esta estrategia y el mal pronóstico de la enfermedad han exigido un replanteamiento de la actitud terapéutica. En este sentido parece oportuna la introducción de fármacos inductores de remisión en las primeras fases de la enfermedad, con la finalidad de reducir la morbilidad y la incapacidad funcional.

Palabras clave: Artritis reumatoide. Tratamiento.
Why an early treatment in rheumatoid arthritis
Key words: Rheumatoid arthritis. Treatment.

\begin{abstract}
Rheumatoid arthritis is an autoimmune disease characterized by chronic inflammation of diarthrodial joints, which may also cause involment of other organs. Today, most authors agree that nearly $80 \%$ of the patients will present with some degreee of disability after 10 years evolution. Prognosis of rheumatoid arthritis is uncertain, and lesions, that are produced in the first years of the disease, may progress despite clinical improvement, since pathogenesis of these lesions may differ from simple acute joint inflammation. Early diagnosis is therefore essential in order to start treatment before structural joint damage develops, since there is evidence that patients treated during the first year from the beginning of symptoms present better functional capacity outcome than those treated later.

Classic treatment for this disease had been based, until recently, on the traditional therapeutic pyramid which consisted in sequential introduction of drugs. Poor results of this strategy and awareness of the bad prognosis of the disease has demanded a reevaluation of its therapeutic approach. In this sense, introduction of remission induction agents early in the course of the disease seems appropiate, with the aim of reducing morbidity and functional disability.
\end{abstract}




\section{INTRODUCCIÓN}

La artritis reumatoide es una enfermedad autoinmune caracterizada por la inflamación crónica de las articulaciones diartrodiales, que además, puede ocasionar afectación de otros órganos (Fig. 1).El deterioro progresivo, articular o extraarticular, acaba provocando deformidad, incapacidad funcional y disminución de la expectativa de vida ${ }^{1}$. Su prevalencia se estima alrededor del $0,5 \%$ en la población española ${ }^{2}$. Ante la sospecha diagnóstica de artritis reumatoide, de forma tradicional los objetivos terapéuticos han sido: reducir la inflamación, preservar la función y prevenir las deformidades, llegándose a considerar que la mayoría de pacientes tenían un buen pronóstico ${ }^{3}$. En la actualidad, la mayoría de autores están de acuerdo en que, a los 10 años de evolución, de cada 100 artritis reumatoides, 10 están en invalidez total, 25 tienen limitaciones importantes, 45 tienen un grado moderado de limitación y sólo 20 mantienen una capacidad funcional normal ${ }^{4}$. Hoy sabemos que el pronóstico de la enfermedad es ciertamente muy variable e impredecible y depende de la instauración de un tratamiento precoz, antes de que aparezcan las lesiones radiológicas 5 .

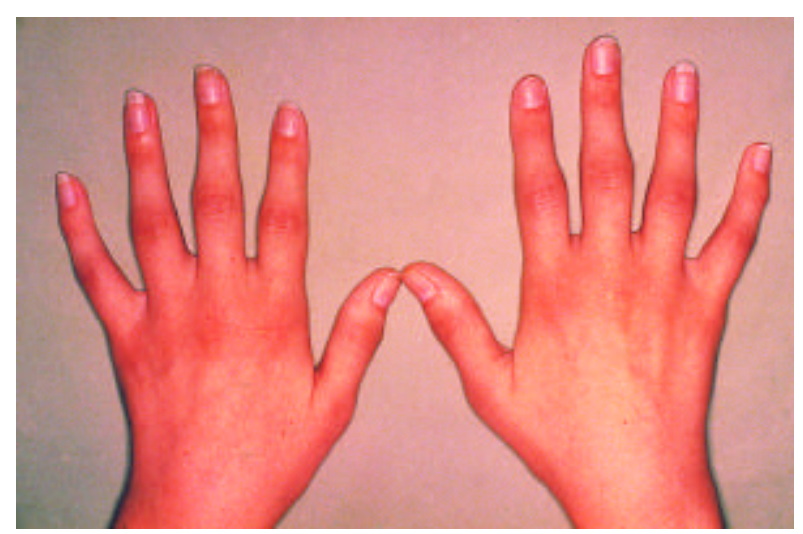

Figura 1

Artritis simétrica de las manos

Hasta ahora la lesión articular se había relacionado con la actividad clínica inflamatoria, pensando que la inflamación o sinovitis aguda era la responsable de la destrucción articular, pero si la inflamación sinovial es la responsable del dolor y la hinchazón local, sabemos que la hiperplasia de la sinovial, la sinovitis crónica, es la responsable de las lesiones articulares 6 , y que es posible que pacientes sin aparente actividad inflamatoria, vayan deteriorando sus articulaciones progresivamente. También se ha relacionado la lesión articular con los distintos patrones evolutivos, de modo que se han caracterizado tres cursos en función de la acti- vidad articular. Uno progresivo (10\%), otro remitente $(20 \%)$ y el más frecuente, intermitente $(70 \%)^{7}$. Sin embargo, con excepción de los casos autolimitados, la enfermedad tiende a presentar un curso progresivo, puesto de manifiesto por la rapidez de las alteraciones radiológicas y la discapacidad. Conviene diferenciar pues entre actividad y gravedad. La actividad se podría definir como la magnitud de la inflamación en un momento dado. Gravedad es un término más complejo y hace referencia a la evolución y al resultado final de la enfermedad, como consecuencia de la suma de la inflamación en un momento determinado y las lesiones acumuladas por la actividad previa ${ }^{8}$. La pregunta es pues, ¿qué relación existe entre la actividad de la enfermedad y las lesiones articulares y sus consecuencias?

\section{RELACIÓN ENTRE ACTIVIDAD CLÍNICA DE LA AR Y LA APARICIÓN DE LESIONES Y DISCAPACIDAD}

La sospecha de que a pesar de la mejoría clínica, las lesiones articulares siguen progresando es un hecho conocido. En 1996 Mulherin publicó una serie de 40 pacientes afectos de artritis reumatoide, seguidos durante 6 años, y en los que observó que a pesar de la mejoría en todos los parámetros de actividad, clínica y biológica monitorizados, la lesión articular progresaba9 ${ }^{9}$ En la figura 2 se observa que aunque se produzcan diferentes grados de mejoría en la fuerza de prensión, VSG, índice articular de Ritchie, rigidez matutina, dolor evaluado por EAV y la tasa de hemoglobina, el índice de lesión articular empeora en estos pacientes. La conclusión es que las erosiones articulares continúan produciéndose en la AR a pesar de la mejoría clínica y se aceleran con la inflamación sinovial, y que su patogénesis, tal como se sospechaba, puede diferir de la simple inflamación.

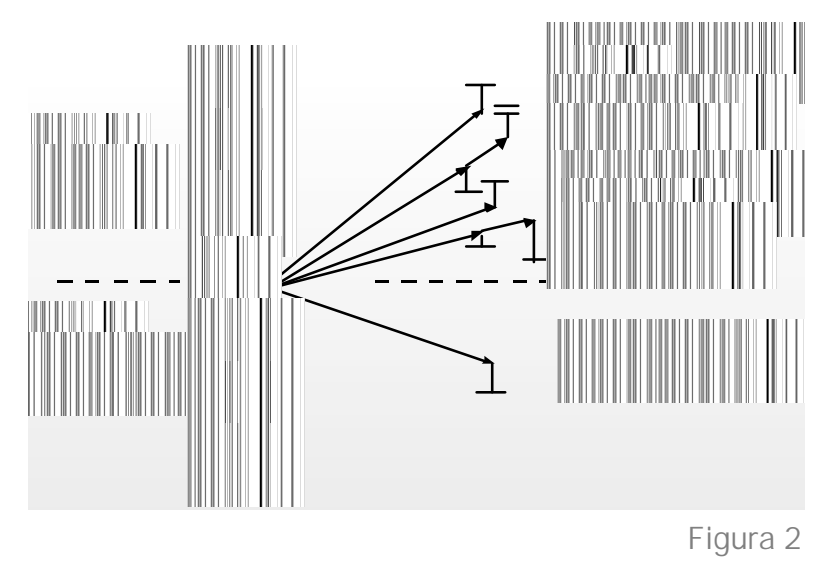

Relación entre mejoría clínica y erosiones en la AR

(Mulherin 1996) 
En 1999 Kirwan publicó una revisión de la literatura con la intención de estudiar la relación existente entre inflamación, lesión y discapacidad en esta enfermedad ${ }^{6}$. En la figura 3 se observa que aunque la inflamación articular pueda ser moderada en el tiempo, por acción del tratamiento, la lesión sigue progresando y paralelamente a ella la discapacidad del paciente con artritis reumatoide. De todos modos esta concordancia entre lesión y discapacidad no se da en los primeros años, en los que parece que la discapacidad es la consecuencia de la propia inflamación articular, o al menos es con ella con quien se correlaciona.

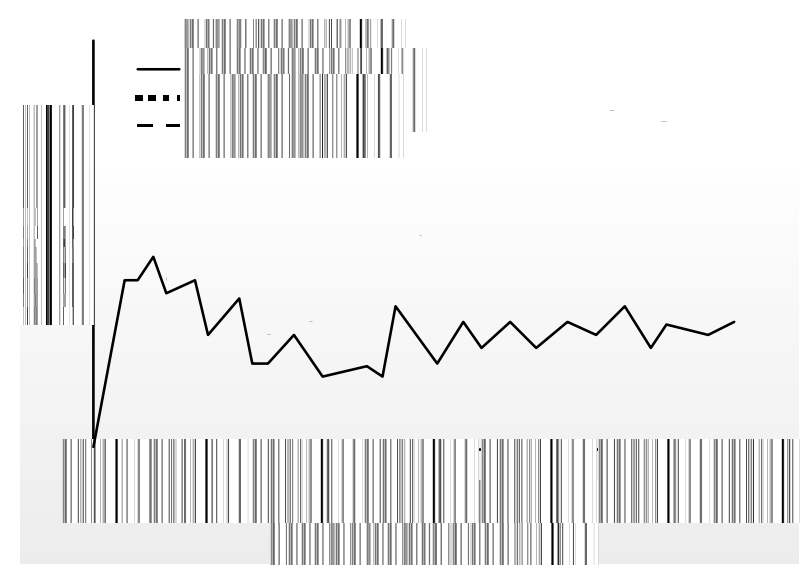

Figura 3

Relación entre inflamación, lesión y discapacidad en la AR (Kirwan 1999).

La relación entre discapacidad y lesión ha sido estudiada por Scott este mismo año, a través de una revisión de más de 50 artículos, observando que en las fases iniciales, la lesión y el HAQ (Health Assessment Questionnary) no se correlacionan, y que es a partir de los primeros 5 años en que se produce una correlación positiva, siendo ésta significativa a partir del $8^{\circ}$ año $(r=0,7)^{10}$. En la figura 4 podemos observar que aunque la lesión articular progresa constantemente con el tiempo, la relación entre lesión y discapacidad sólo se da por encima de los 5 años de evolución.

Hoy sabemos que, en un contexto genético apropiado - la susceptibilidad a la enfermedad se ha asociado al polimorfismo de la región HLA-DR ${ }^{11}$ - un factor o factores iniciadores, hasta ahora desconocidos y con capacidad artritogénica, serían los que desencadenarían la patogenia de esta enfermedad. La presentación de estos antígenos críticos a los linfocitos T CD4 estimularía la expresión de citocinas y de los linfocitos $\mathrm{B}$ con la consiguiente producción de anticuerpos. Los linfocitos T serían de alguna manera los responsables de la inflamación sinovial aguda.

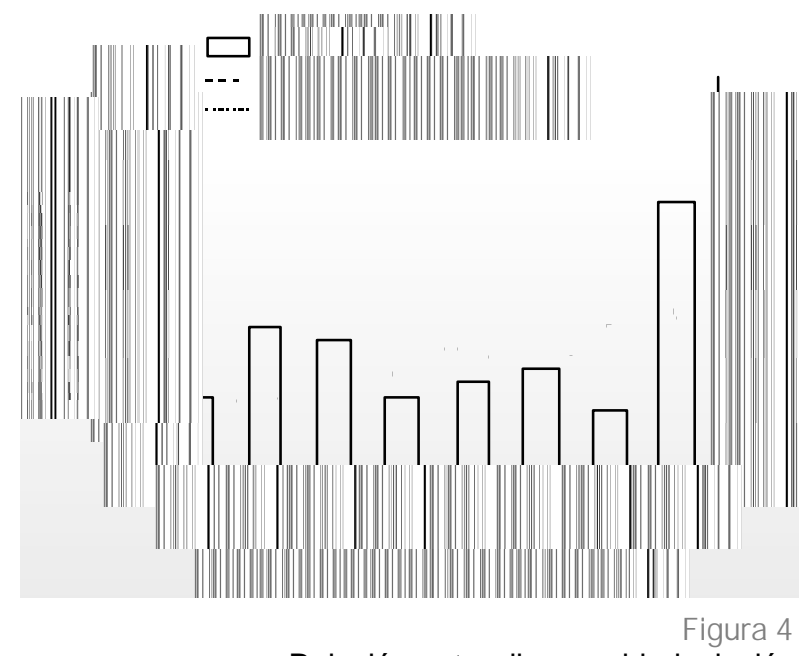

Relación entre discapacidad y lesión

En el pannus sinovial -sinovitis crónica-, las citocinas proinflamatorias predominantes son la IL-1, TNFa, IL-6 y GMCSF, que son producidas por los fibroblastos y macrófagos. La teoría es pues, que son las células de la estirpe monocito-macrófago, más que los linfocitos T, las fundamentales en el proceso inflamatorio crónico. Estas citocinas son capitales en la artritis reumatoide para que se produzca la inflamación y la destrucción articular ya que por un lado estimulan la producción de moléculas de adhesión y por otro inducen la producción de enzimas proteolíticas responsables de la des trucción del cartílago y del hueso ${ }^{12}$. Es decir, los macrófagos y fibroblastos sinoviales, pero no los linfocitos, se correlacionarían con el curso radiológico de la artritis reumatoide, mientras que los linfocitos y otras poblaciones celulares se correlacionarían con las medidas de actividad.

Conocemos también que esta lesión articular en la artritis reumatoide es precoz, y que se produce en los dos primeros años de evolución. La radiología directa, la gammagrafía ósea, la tomografía axial computerizada y la resonancia nuclear magnética, son las técnicas más utilizadas para el estudio de estas lesiones. Aun así, la radiología ósea es la exploración más utilizada, a pesar de que su sensibilidad es más tardía, y se han creado diferentes índices radiológicos para su cuantificación y seguimiento evolutivo como son el de Sharp ${ }^{13}$, Lar$\operatorname{sen}^{14}$, y van der Heijde ${ }^{15}$.

Un tratamiento precoz y agresivo en la artritis reumatoide es el medio más eficaz para minimizar las repercusiones que se derivan de no tratar o tratar inadecuadamente esta enfermedad. Cada vez existen más evidencias que indican la necesidad de iniciar un tratamiento agresivo con fármacos inductores de remisión, en las fases precoces de la enfermedad, demostrando que el retraso en la instauración del tratamiento de fondo influye en su pronóstico a largo plazo ${ }^{16-18}$. 
Recientemente han aparecido en la literatura diversos ensayos clínicos controlados, randomizados y "doble ciego", en los que se recomienda utilizar la combinación de varios de estos fármacos de segunda línea en las fases iniciales de la enfermedad ${ }^{19-20}$. En este sentido, en enero del 2000 ha sido publicado un artículo que evalúa, a partir de un metaanálisis de 14 ensayos clínicos, "doble ciego" y aleatorizados, con un total de 1.435 pacientes, los factores sociodemográficos y de actividad de la enfermedad que pueden afectar a la respuesta del tratamiento ${ }^{21}$.

Teniendo en cuenta que la respuesta al tratamiento debe considerarse como un factor pronóstico, las conclusiones de este trabajo revelan que el sexo femenino, una marcada discapacidad funcional (clase III de la ACR), el uso previo de terapia inductora, una baja actividad clínica y sobre todo, la duración de la artritis, son los factores predictivos independientes de mala respuesta al tratamiento inductor de remisión. Dicho trabajo muestra que todos los tratamientos activos son eficaces con una tasa de respuesta (ACR 20\%) del 53\% para una evolución de la enfermedad inferior a un año, del $38 \%$ entre 5 y 10 años y del $35 \%$ cuando la evolución supera los 10 años. Por todo ello, y hasta que futuras investigaciones en la artritis reumatoide permitan establecer marcadores de pronóstico fiables en las primeras fases, en la práctica clínica diaria sería conveniente iniciar el tratamiento de forma precoz y con fármacos inductores de remisión como estrategia terapéutica de inicio.

En resumen, el pronóstico de la artritis reumatoide es casi siempre incierto, y las lesiones, que se producen en los primeros años de enfermedad, pueden continuar a pesar de la mejoría clínica. La patogénesis de estas lesiones puede diferir de la simple inflamación aguda articular y la relación entre discapacidad y lesión no es uniforme en el tiempo. Lamentablemente, la radiología, a pesar de su baja sensibilidad al inicio de la lesión, es la técnica diagnóstica más eficiente. El diagnóstico precoz es pues esencial para iniciar su tratamiento antes de que se produzca el daño anatómico articular. Todo ello ha llevado a modificar el esquema terapéutico, en el sentido de actuar con fármacos inductores de remisión en las primeras fases de la enfermedad, con la finalidad de reducir la morbilidad y la incapacidad funcional.

\section{FACTORES PRONÓSTICOS EN LA ARTRITIS REUMATOIDE DE COMIENZO RECIENTE}

El diagnóstico precoz es el factor pronóstico más importante. Es por ello que cada vez existen más consultas destinadas a atender a las artritis de comienzo reciente. Se ha demostrado que los pacientes referidos a una consulta de Reumatología durante el primer año desde el comienzo de los síntomas, mantienen una capacidad funcional mejor que los remitidos más tardíamente. También se ha demostrado que los enfermos no tratados de forma específica tienen una mayor discapacidad ${ }^{22}$, y que la duración de la enfermedad es el factor más importante que se correlaciona con las lesiones radiológicas. Así, se han observado erosiones en un $37 \%$ de pacientes con artritis de inicio de 3 a 4 meses de evolución, alcanzando el $91 \%$ en los pacientes diagnosticados a los 36 meses $^{23}$. $\mathrm{Y}$ en un estudio de cohortes publicado recientemente por Sokka se pone de manifiesto que existe una menor discapacidad funcional cuando se inicia el tratamiento de forma precoz ${ }^{24}$. Así pues, para establecer un diagnóstico y tratamiento precoz de esta enfermedad será necesario consensuar por parte de los reumatólogos, los criterios de diagnóstico precoz de la artritis reumatoide, así como establecer programas de difusión social y de divulgación en el conocimiento básico de la semiología y patología reumatológica en la Atención Primaria, con el fin de sensibilizar al máximo y colaborar con los médicos de asistencia primaria en el estudio, diagnóstico y tratamiento precoz del síndrome clínico "artritis de inicio" ello, que cada vez existen más consultas destinadas a atender las artritis de comienzo reciente. En ellas se debe contemplar la sospecha de artritis en todos los pacientes que acuden por dolor, inflamación articular y/o limitación de la movilidad, sin antecedente traumático. En el caso concreto de la artritis reumatoide, los criterios de clasificación de la ACR de $1987^{25}$, no permiten establecer, por su baja sensibilidad, un diagnóstico de inicio de la enfermedad. Es por ello que se han estudiado muchos factores de inicio en relación al pronóstico de la enfermedad ${ }^{26}$, y es probable que la combinación de ellos puedan ayudar a intuirlo (Tabla I), pudiendo afirmar, en función de diversos estudios, que entre el $70-80 \%$ de pacientes, es decir, aproximadamente 3 de cada 4 pueden llegar a ser

Tabla I

FACTORES PREDICTIVOS EN LA ARTRITIS LIGADOS AL PACIENTE

Características socioeconómicas y culturales

Sexo femenino

Estado psicológico

Características inmunogenéticas

LIGADOS A LA ENFERMEDAD

Contaje articular (dolor e inflamación) alto

Persistencia de sinovitis tras el tratamiento

Erosiones articulares precoces

Disminución de la capacidad funcional

Elevación de la VSG y la PCR

Afectación extraarticular (nódulos, vasculitis, anemia, etc.)

Modificado de Alarcón GS, 1997 
identificados como de mal pronóstico ${ }^{27}$.

\section{NUEVAS ESTRATEGIAS TERAPÉUTICAS} EN LA ARTRITIS REUMATOIDE

El tratamiento clásico de esta enfermedad había contemplado, hasta hace poco tiempo, una estructura piramidal, en la que de forma secuencial se iban abordando, en primer lugar el programa básico, seguido en una segunda fase de la administración de fármacos modificadores de la enfermedad, de la utilización de corticosteroides en la tercera fase y finalmente de la utilización de fármacos inmunosupresores, a medida que la artritis reumatoide iba empeorando. El programa básico, que incluye la información al paciente y su entorno, el apoyo social, la fisioterapia y los antiinflamatorios no esteroideos, a pesar de que puede proporcionar una mejoría sintomática, no modifica la evolución natural de la enfermedad. Los fármacos modificadores de la enfermedad o inductores de remisión, tal como las sales de oro, los antipalúdicos, la d-penicilamina, la salazopirina, y el metotrexato, tienen unas características comunes: no se conoce exactamente su mecanismo de acción, no se puede preveer su grado de eficacia, tardan entre 6 y 12 semanas en actuar, exigen su administración continuada y en más de la tercera parte de los pacientes, las reacciones adversas obligan a su suspensión. Los corticosteroides, que a dosis bajas producen una rápida acción sintomática han sido siempre objeto de polémica, siendo reservados para los casos refractarios al tratamiento o con manifestaciones extraarticulares graves. Finalmente, los inmunosupresores, como la azatioprina, ciclofosfamida, cloranbucilo y ciclosporina A, quedaban restringidos a aquellos enfermos que no respondían a la anterior escalada terapéutica y en los que el proceso continuaba siendo suficientemente activo. Con este programa terapéutico, no era raro que los fármacos inductores de remisión fueran introducidos tras varios años de evolución, cuando las lesiones, y la discapacidad estaban ya instauradas, y a pesar de que todos ellos han mostrado su eficacia en estudios controlados, se hace difícil conocer su efecto real sobre la enfermedad, y sólo un $20 \%$ de pacientes siguen tomando el fármaco a los 5 años ${ }^{28}$.

Los pobres resultados obtenidos con el tratamiento clásico y su mal pronóstico han exigido un replanteamiento de la actitud terapéutica. En 1995, el Colegio Americano de Reumatología, ya contemplaba en su guía terapéutica, la introducción de corticosteroides, a pequeñas dosis, en la primera línea farmacológica ${ }^{29}$, y en los últimos años se han utilizado terapias combinadas de estos fármacos. Las asociaciones de metotrexato como fármaco de base, asociado a hidroxicloroquina y sulfasalazina ${ }^{30}$, o a ciclosporina $\mathrm{A}^{31}$, se han mostrado efectivas, aunque no existe una indicación clara sobre el tipo de pacientes que debieran recibirla cuando está indicada, ni cuál es la combinación ideal de fármacos a emplear.

Recientemente han aparecido nuevos fármacos inductores de remisión. La leflunomida, un derivado isoxazólico de bajo peso molecular, es un inmunorregulador no relacionado estructuralmente con ningún otro inmunosupresor ${ }^{32}$. Este fármaco ha demostrado mejorar los parámetros clínicos de la artritis reumatoide, y los indicadores de calidad de vida, siendo también capaz de retrasar significativamente la progresión de las lesiones radiológi$\operatorname{cas}^{33-36}$. El infliximab y el etanercept, son anticuerpos monoclonales que interfieren el TNFa humano, neutralizando su actividad biológica, esencial en la patogenia de la enfermedad. Ambos han demostrado su eficacia en el control de las manifestaciones clínicas, con una buena tolerancia ${ }^{37-43}$.

Si bien las recomendaciones sobre el uso de uno u otro de estos fármacos dependen de cada situación clínica particular y, sobre todo, de la experiencia de cada reumatólogo, existen una serie de parámetros clínicos y analíticos que permiten proponer una orientación terapéutica en el uso secuencial de

\section{Tabla II}

NIVELES DE TRATAMIENTO EN LA ARTRITIS

NIVEL 1. AR CLÍNICAMENTE LEVE, SIN EROSIONES RADIOLÓGICAS
Oro oral 0
Antimaláricos 0
Salazopirina

NIVEL 2. AR CON FACTORES DE MAL PRONÓSTICO Y/O EROSIONES
Metotrexato 0
Leflunomida 0
Oro parental 0
D-penicilamina
Terapias anti-TNF 0
Ciclosporina A?

NIVEL 3. AR AGRESIVA REFRACTARIA AL NIVEL 2
Combinaciones de probada eficacia
Metotrexato + Ciclosporina A
Metotrexato + Cloroquina + Salazopirina
Metotrexato + Cloroquina
Prednisona + Metotrexato + Salazopirina
Terapias anti-TNF (solas o con metotrexato)

NIVEL 4. SITUACIONES ESPECIALES COMO AMILOIDOSIS, AR REFRACTARIAS A LOS OTROS NIVELES, SITUACIONES DE RIESGO VITAL

Terapias experimentales: TMO

Terapias de alta toxicidad

TMO: trasplante de médula ósea (Queiro-Silva y Riestra Noriega 
ellos $^{44}$, como se muestra en la tabla II.

Los objetivos del tratamiento de la artritis reumatoide, a nivel articular, siguen siendo reducir la inflamación, preservar la función y prevenir las deformidades. Hemos visto que la patogénesis de estas lesiones difiere de la simple inflamación articular, y que la lesión se produce en los primeros años de la enfermedad. El diagnóstico precoz va a ser pues esencial para la instauración de un tratamiento más activo que impida el desarrollo del daño articular. En este sentido las aptitudes y actitudes de los médicos de Atención Primaria y de los reumatólogos, así como su coordinación al inicio del

\section{CORRESPONDENCIA:}

P. Benito Ruiz

Servicio de Reumatología

Hospital Universitario del Mar

Paseo Marítimo, 25-29

08003 Barcelona

Tel. 932211010 - Fax 932211876

E-mail: 17047m@imas.imim.es

pbenito@imas.imim.es

\section{Bibliografía}

1. Laffon-Roca A, Ortiz-García AM. Etiopatogenia y epidemiología de la artritis reumatoide. En: Alonso-Ruiz A. Manual SER de las Enfermedades Reumáticas. $3^{\text {a }}$ Ed Panamericana 2000; 257-63.

2. SER. EPISER 2000. Prevalencia de la artritis reumatoide, lumbalgia, artrosis de rodillas y manos, fibromialgia, osteoporosis y lupus eritematoso sistémico en la población española 2000. Med Clin (en prensa).

3. Hilliquin P, Menkes Ch-J. Evaluation and management in rheumatoid arthritis: early and established disease. En: Klippel J, Diepe PA. Mosby Rheumatology 1994; 3.13.1-3.13.14.

4. Muñoz Gómez J. Artritis reumatoidea. En: Rotés Querol J. Expaxs Reumatología Clínica 1983; 75-98.

5. Batlle-Gualda E. Artritis reumatoide grave. Métodos para medir la actividad de la artritis. Criterios pronósticos y su relación con el tratamiento. En: Rodriguez de la Serna A. Nuevas terapéuticas en la artritis reumatoide. Barcelona: Ed MCR 1996; 17-32.

6. Kirwan JR. Conceptual Issues in Scoring Radiographic Progesssion in Rheumatoid Arthritis. J Rheumatol 1999; 26 (3): 720-5.

7. Masi AT, Feigenbaum SL, Kaplan SB. Articular patterns in the early course of rheumatoid arthritis. Clinical, synovial fluid and biopsy studies. Arthritis Rheum 1982; 25: 361-9.

8. Martínez A, Batlle-Gualda E, Pascual E. Curso y evolución de la artritis reumatoide. Seminarios de la Fundación Española de Reumatología 2000; 2: 65-72.

9. Mulherin D, Fitzgerald O, Bresnihan B. Clinical improvement and radiological deterioration in RA: evidence that the patogenesis of synovial inflammation and articular erosion may differ. $\mathrm{Br} \mathrm{J}$ Rheumatol 1996; 35: 1263-8.

10. Scott DL, Pugner K, Kaarela K, Doyle DV, Wolf S, Holenes $J$ et al. The links between joint damage and disability in rheumatoid arthritis. Rheumatology 2000 ; 39: 122-32.

11. Weyand CM, Hicok KC, Conn D, Gorozny JJ. The influence of HLA-DRB1 genes on disease severity in rheumatoid arthritis. Ann Inter Med 1992; 117: 801-6.

12. Bresnihan B. Pathogenesis of joint damage in rheumatoid arthritis. J Rheumatol 1999; 26: 717-9.

13. Sharp JT. Scoring radiographic abnormalities in rheumatoid arthritis. J Rheumatol 1989; 16: 568-9.
14. Larsen A, Dale K, Eek M. Radiographic evaluation of rheumatoid arthritis and related conditions by standard reference films. Acta Radiol Diagn 1977; 18: 481-91.

15. Van der Heijde D. How to read radiographs according to the Sharp/van der Heijde method. J Rheumatol 1999; 26: 740-2.

16. Tsakonas E, Fitgerald AA, Fitzcharles MA, Ciridino A, Thorne JC, M'Seffar A, et al. Consequences of delayed therapy with second-line agents in rheumatoid arthritis: a 3 year followup on the hydroxychloroquine in early rheumatoid arthritis (HERA) study. J Rheumatol 2000; 27 (3): 623-92.

17. Egsmose C, Lund B, Borg G, Petterson H, Berg E, Brodin V, et al. Patients with rheumatoid arthritis benefit from early 2 nd line therapy: 5 year followup of a prospective double blind placebo controlled study. $J$ Rheumatol 1995; 22: 2208-13.

18. Lard IR, Visser H, Speyer I, van der Horst-Bruinsma $\mathrm{IE}$, Zwinderman AH. Early vs delayed treatment in recent onset rheumatoid arthritis patients: results from a two year controlled followup study in an early arthritis clinic. Arthritis Rheum 2000; 43 (9 Suppl): S154.

19. Mottonen TT, Hannonen Pj, Boers M. Combination DMARD therapy including corticosteroids in early rheumatoid arthritis. Clin Exp Rheumatol 1999; 17 (6 Suppl 18): S59-65.

20. Dougados M, Combe B, Cantagrel A, Goupille P, Olive $P$, Schatteinkirchner $M$, et al. Combination therapy in early rheumatoid arthritis: a randomised, controlled, double blind 52 week clinical trial of sulphasalazine and methotrexate compared with the single components. Ann Rheum Dis 1999; 58 (4): 220-5.

21. Anderson JJ, Wells G, Verhoven AC, Felson DT. Factors predicting response to treatment in rheumatoid arthritis: the importance of disease duration. Arthritis Rheum 2000; 43 (1): 22-9.

22. Ward MM, Leigh JP, Fries JF. Progression or functional disability in patients with rheumatoid arthritis. Associations with rheumatology subspeciality care. Arch Intern Med 1993; 153: 2229-37.

23. Caruso I, Santandrea S, Sarzi Puttini P, Boccasini L, Montsone F, Cazzola M, et al. Clinical, laboratory and radiogra-phic features in early rheumatoid arthritis. $\mathrm{J}$ Rheumatol 1990; 17 (10): 1263-7. 
24. Sokka T, Mottonen T, Hannonen P. Disease-modifying anti-rheumatic drugs use according to the "sawtooh" tratment strategy improves the functional outcome in rheumatoid ar-thritis: results of a long-term follow-up study with review of the literature. Rheumatology (Oxford) 2000; 39 (1): 34-42.

25. Arnett FC, Edworthy S, Block DA, Mc Shane DJ, Fries JF, Cooper NS, et al. The 1987 revised ARA criteria for rheumatoid arthritis. Arthritis Rheum 1987; 30: S17.

26. Alarcon GS. Predictive factors in rheumatoid arthritis. Am J Med 1997; 103 (6A): 19S-24S.

27. Sanmartí Sala R. ¿Se puede predecir la evolución de la artritis reumatoide? Rev Esp Reumatol 2000; 27 (6): $260-5$

28. Rodriguez-Valverde $V$, Fariñas-Alvarez C. ¿Es eficaz el tratamiento actual de la artritis reumatoide? Mec Clin (Barc). 1990; 94: 499-501.

29. Guidelines for the management of rheumatoid arthritis. Arthritis Rheum 1996; 39 (5): 713-22.

30. O'Dell R, Haire C, Erikson N, Drymalski N, Palmer W, Eckhoff PJ, et al. Treatment of rheumatoid arthritis with methotrexate alone, sulfasalazine and hydroxychloroquine, or a combination of all three medications. New Engl J med 1996; 334: 1287-91.

31. Tugwell $P$, Pincus $T$, Yocum D, Stein M, Gluck $O$, Kraag G, et al. Combination therapy with cyclosporine and methotrexate in severe rheumatoid arthritis. New Engl J med 1995; 333: 137-41.

32. Fox RI, Herrmann ML, Frangou CG, Wahl GM, Morris RE, Strand V, et al. Mechanism of action for Leflunomide in rheumatoid arthritis. Clinical Inmunology 1999; 93 (3): 198-202.

33. Sharp JT, Strand V, Leung H, Hurley F, Loew-Friedrich L. Treatment with Leflunomide slows radiographic pro- gression of rheumatoid arthritis. Artritis Rheum 2000; 43 (3): 495-505

34. Strand V, Tugwell $P$, Bombardier $C$, Maetzel A, Crawford B, Dorrier C, et al. Function and Health-related quality of life. Arthritis Rheum 1999; 42 (9): 1870-8.

35. Smolen JS, Kalden JR, Scott DL, Rozman B, Kvien TK, Larsen A, et al. Efficacy and safety of leflunomide compared with placebo and sulphasalazine in active rheumatoid arthritis: a double-blind, randomised, multicentre trial. Lancet 1999; 353 (9149): 259-66.

36. Emery P, Breedveld C, Lemmel EM, Kaltwasser JP, Dawesp PT, Gomor B, et al. A comparison of the efficacy and safety of leflunomide and methotrexate for the treatment of rheumatoid arthritis. $\mathrm{Br} \mathrm{J}$ Rheumatol $2000 ; 655-64$

37. Rodríguez Valverde V, Martínez Taboada V, Blanco Alonso R. Leflunomida en la artritis reumatoide. Mecanismos de acción, eficacia y toxicidad. Seminarios de la Fundación Española de Reumatología 2000; 1 (2): 73-82.

38. Alvaro-Gracia JM, Ortiz García A. Tratamiento con Infliximab en la artritis reumatoide. Rev Esp Reumatol 2000; 27 (6): 293-5.

39. Maini R, St Clair EW, Breedveld F, Furst D, Kalden J, Weisman M, et al. Infliximab (chimeric anti-tumor necrosis factor monoclonal antibody) versus placebo in rheumatoid arthritis patients receiving concomitant methotrexate: a randomised phase III trial Lancet 1999; 354 (9194): 1932-9.

40. Lipsky PE, van der Heijde D, St Clair WS, Furst DE, Breedveld FC, Kalden JR, et al. Infliximab and methotrexate in the treatment of rheumatoid arthritis. N Engl J Med 2000; 343 (22): 1594-602.

41. Weinblatt ME, Kremer JM, Bankurst AD, Bulpitt KJ, Fleishmann RM, Fox RI, et al. A trial of etanercept, a 